\title{
ASSISTÊNCIA À CRIANÇA COM CÂNCER: ALGUNS ELEMENTOS PARA A ANÁLISE DO PROCESSO DE TRABALHO
}

\author{
Regina Aparecida Garcia de Lima* \\ Carmem Gracinda Silvan Scoch $i^{*}$ \\ Ivone Kamada* \\ Semíramis Melani Melo Rocha*
}

LIMA, R.A.G. de et al. Assistência à criança com câncer: análise do processo de trabalho. Rev.Esc.Enf.USP, v. 30, n.1, p.14-24, abr. 1996.

Este estudo procura analisar alguns elementos do processo de trabalho na assistência à criança com câncer, fundamentado no Modelo de Organização Tecnológica do Trabalho. Partiu-se do princípio teórico de que a enfermagem é uma prática social e, portanto, historicamente estruturada e socialmente articulada; desta forma a enfermagem é parte do processo coletivo de trabalho em saúde. Apontamos para o fato de que o processo de trabalho da enfermagem na assistência à criança com câncer está em fase de transformação, passando do modelo exclusivamente clínico para outro, que tem como características a ampliação do referencial teórico e nova instrumentalização.

UNITERMOS: Neoplasia-criança. Assistência de Enfermagem.

\section{INTRODUÇÃO}

A análise dos dados nosológicos e de mortalidade tem demonstrado que o câncer é um relevante problema de saúde coletiva no Brasil e a nível mundial ${ }^{12}$. O Brasil apresenta um quadro sanitário onde as chamadas doenças de sociedades desenvolvidas (doenças do aparelho circulatório e neoplasmas) e as doenças associadas ao subdesenvolvimento (infecciosas e parasitárias) combinam-se e quanto as causas de bbitos, as primeiras são mais comuns nas regiões Sul e Sudeste, enquanto as últimas no Norte e Nordeste?

* Professores do Departamento de Enfermagem Materno-Infantil e Saúde Pública da Escola de Enfermagem de Ribeirão Preto-USP, Grupo de Estudos em Saúde da Criança e do Adolescente. 
O câncer já representa a terceira causa isolada de morte no Brasil, e a segunda em alguns estados e até meados da década de 90 estima-se uma ocorrência anual de aproximadamente 200 mil casos e 90 mil obitos por esta patologia ${ }^{6}$.

A incidência das neoplasias malignas em crianças não é tão alta como em adultos, porém, no Brasil e em outros países, constitui-se uma das causas de morte mais freqüente e, se eliminadas outras causas, como infecção e desnutrição, as neoplasias passam a constituir um problema de saúde, especialmente no grupo de 1 a 5 anos de idade 1 . O câncer infantil atinge 10 em cada 100 mil crianças a cada ano em todo o mundo, sendo que uma criança em cada 600 pode desenvolvê-lo durante a infância. Na atualidade $2 / 3$ destes cânceres podem ser considerados curáveis se a terapêutica instiuida for adequada ${ }^{4}{ }^{15}$.

A hospitalização da criança com câncer tem características diversas das demais em uma unidade de internação. As mais significativas são a possibilidade de um mau prognóstico, a cronicidade da doença, a duração e as reinternações freqüentes, os traumas físicos e psíquicos, a terapêutica agressiva, a alteração da auto-imagem, a alta mortalidade, somando-se ainda problemas de ordem familiar provocado pelo diagnóstico.

A assistência de enfermagem prestada a estas crianças, geralmente, tem por base uma série de técnicas referentes a higiene, alimentação, colheita de material para exames e administração de medicação. Na maioria das vezes estes cuidados atendem apenas aos aspectos do corpo biológico, não considerando esta criança como um ser em crescimento e desenvolvimento, com determinações familiares, culturais, ambientais e econômicas ${ }^{11}$.

A insatisfação com este tipo de trabalho motivou a equipe de saúde da Clínica Pediátrica do Hospital das Clínicas da Faculdade de Medicina de Ribeirão Preto da Universidade de São Paulo a estruturar um grupo multiprofissional para reorganizar a assistência à criança com câncer.

Esta equipe iniciou suas atividades em 1984 e em 1986 criou o GRUPO DE APOIO Â CRIANÇA COM CÂNCER - GACC, entidade civil, sem fins lucrativos, que tem como finalidade apoio integral, de ordem bio-psico-social à criança portadora de câncer e a sua família. Deu-se desta forma a institucionalização da equipe.

Dentro da nova abordagem proposta pela equipe multiprofissional, o processo de trabalho da enfermagem tornou-se mais complexo. Alem dos instrumentos que possibilitavam o desempenho das técnicas de enfermagem para atender o ato médico centrado na patologia, a enfermagem passou a des envolver ações que auxiliavam no relacionamento da criança com a família, da criança com a equipe e da criança com a equipe e a família. As questões afetivas, emocionais, psicologicas e sociais foram trazidas para o hospital e passaram a ter significado, pois fazem parte do processo de desenvolvimento 
da criança. O objetivo da assistência passou a ser proporcionar uma melhor qualidade de vida, levando em consideração a integridade da criança, em toda sua especificidade.

Para responder a esta nova necessidade, os instrumentos ou meios de trabalho também alteram-se. Novas habilidades, ações e abordagens de enfermagem são acrescentadas as já existentes, utilizando-se outros conhecimentos além da Anatomia, Fisiologia e Patologia. A enfermagem vai buscar no referencial da Psicologia, Epidemiologia, Sociologia e Antropologia, conhecimentos para atender as necessidades dessa criança doente e sua familia.

\section{Objetivo}

O objetivo principal desta pesquisa 6 analisar o processo de trabalho na assistência de enfermagem à criança com câncer na sua historicidade e socialidade.

Para tanto, procedemos a caracterização da criança com câncer atendida no Hospital das Clínicas da Faculdade de Medicina de Ribeirão Preto-USP, levando em consideração as variáveis: sexo, idade, tipo de câncer, procedência e terapêutica. A seguir, analisaremos a assistência de enfermagem enfocando as transformações no processo de trabalho tendo por base algumas ações específicas da enfermagem.

\section{Referencial Teórico}

Utilizaremos o Modelo de Organização Tecnológica do Trabalho proposto por GONÇALVES ${ }^{10}$ e desenvolvido a partir da Teoria do Trabalho, concebida por Marx, como referencial teórico para a nálise do processo de trabalho da enfermagem na assistência à criança com câncer.

São três os elementos essenciais do processo de trabalho: a atividade orientada para uma finalidade ou o próprio trabalho, seu objeto e seus meios ou instrumentos. Assim, um objeto de trabalho pode ser definido como um fragmento da realidade, recortado por um olhar que concebe intelectualmente um produto com qualidades e potencialidades de transformar-se nesse produto. Os instrumentos ou meios de trabalho permitem a aproximação e transformação do objeto, sendo que as caracteristicas do objeto, de um lado e a finalidade do trabalho, de outro, determinam as caracteristicas dos meios de trabalho ${ }^{10}$. A separação entre objeto, instrumento e produto só é possível abstratamento, pois todos são momentos interdependentes do processo de trabalho. 
Tratando especificamente do Processo de Trabalho em Saúde, e este, nas sociedades capitalistas, GONCALVES ${ }^{10}$ aponta dois modelos principais de organização das práticas de saúde: o modelo de controle da doença na sociedade, que é o modelo epidemiológico, coletivo e o modelo de recuperação da força de trabalho, que é o individual, o clínico.

A concepção da saúde e da doença dada pela clínica está inteiramente contida nos limites fisícos, químicos e biologicos do corpo humano. Esta forma de pensar o corpo é definida a partir da Anatomia, Fisiologia e Patologia. Assim, neste modelo, a assistência a saúde pauta-se principalmente na patologia e nos sinais e sintomas; um grande esforço é feito no sentido de conseguir um diagnóstico acurado e pronta instalação de medidas terapêuticas.

Já o conhecimento da saúde e da doença em sua dimensão coletiva acha-se estruturada basicam ente na Epidemiologia e esta apreende a saúde e a doença nos corpos enquanto coletivo, es tabelecendo relação entre o coletivo e o meio ${ }^{10}$. Outras dimensões da saúde e da doença, enquanto fenômenos coletivos, acham-se também trabalhadas e acumuladas nas ciências sociais, principalmente na Sociologia, Antropologia, Economia e na Ciência Política.

\section{MATERIAL E MÉTODO}

O levantamento de dados* foi realizado no Hospital das Clínicas da Faculdade de Medicina de Ribeirão Preto da Universidade de São Paulo HCFMRP-USP.

Esta instituição é um hospital-escola, que tem por finalidade o ensino, a pesquisa e a extensão de serviços à comunidade. É um hospital de referência para o SUS (ERSA-50)que comporta 22 municípios, abrangendo uma área de $9.398 \mathrm{Km}^{2}$ e com uma população estimada para 1987 de 812.772 habitantes ${ }^{14}$, recebendo também pacientes de outras regiões e estados.

A população de referência foi composta por pacientes menores de 15 anos de idade, com diagnóstico de câncer, atendidos no HCFMRP-USP. Desta população selecionamos aqueles atendidos na Clínica Pediátrica e que estiveram internados no período de $1^{\circ}$ de janeiro a 31 de dezembro de 1987. Consideramos a nomenclatura da Classificação Internacional de Doenças ${ }^{8}$ para a identificação dos cânceres.

\footnotetext{
* LIMA, R.A.G.; ROCHA, S.M.M.; TONE, L.G. O câncer infantil: caracterização da população atendida em um hospital - escola como subsídios para a assistência de enfermagem. Medicina, v.26, n.4, p.627.635, 1993.
} 
A relação dos pacientes foi fornecida pelo Serviço de Arquivo Médico e Estatística (SAME). A coleta de dados foi feita a partir da análise retrospectiva dos prontuários destes pacientes e as seguintes variáveis foram estudadas: sexo, idade, tipo de câncer, procedência e terapêutica. Foram rigorosamente acatados os diagnósticos definitivos registrados nos prontuários, independentemente da comprovação histopatológica.

\section{RESULTADOS E DISCUSSÃO}

Foram atendidos 62 pacientes, menores de 15 anos; com diagnóstico de câncer, no período de 1 de janeiro a 31 de dezembro de 1987, no Hospital das Clínicas da Faculdade de Medicina de Ribeirão Preto-USP. Destes, 39 (62,9\%) estiveram internados na Clinica Pediátrica e dado nosso interesse foram objeto de estudo. Os dados por nós obtidos são de freqüência, limitando esta investigação, uma vez que os coeficientes de incidência, prevalência e mortalidade não serão discutidos. Trata-se, porém, de um estudo preliminar relativo à assistência de enfermagem e não sobre a epidemiologia da doença.

A seguir demonstraremos os resultados obtidos pela caracterização destes pacientes, iniciando com o diagnóstico e a faixa etária, conforme Tabela

I. A idade foi obtida considerando a época do diagnóstico.

TABELA I. Distribuição do diagnóstico de câncer em crianças menores de 15 anos de idade, internadas na Clínica Pediátrica do HCFMRP-USP, segundo faixa etária, no periodo de 01/01 a 31/12/87.

\begin{tabular}{lcccccccc}
\hline \multirow{2}{*}{ Faixa Etáriai } & $01 \vdash$ & 5 & $5 \vdash$ & 10 & $10 \vdash$ & \multicolumn{2}{c}{ T o t a 1} \\
\cline { 2 - 9 } Diagnóstico & $\mathbf{n}^{\circ}$ & $\%$ & $\mathbf{n}^{\circ}$ & $\%$ & $\mathbf{n}^{\circ}$ & $\%$ & $\mathbf{n}^{\circ}$ & $\%$ \\
\hline Leucemia Linfóide Aguda (LLa) & 7 & 17,9 & 5 & 12,8 & 1 & 2,5 & 13 & 33,3 \\
Leucemia Mielóide Aguda (LMA) & 2 & 5,1 & 4 & 10,2 & 2 & 5,1 & 8 & 20,5 \\
Leucemia Mielóide Crônica (LMC) & - & - & 1 & 2,5 & - & - & 1 & 2,5 \\
Doença de Hodgkin & 1 & 2,5 & - & - & 2 & 5,1 & 3 & 7,6 \\
Rabdomiossarcoma & - & - & 3 & 7,6 & - & - & 3 & 7,6 \\
Sarcoma de Ewing & 2 & 5,1 & - & - & - & - & 2 & 5,1 \\
Neuroblastoma & - & - & 2 & 5,1 & - & - & 2 & 5,1 \\
Hepatoblastoma & 1 & 2,5 & 1 & 2,5 & - & - & 2 & 5,1 \\
Retinoblastoma & 1 & 2,5 & - & - & - & - & - & 2,5 \\
Tumor de Ovário & - & - & - & - & 1 & 2,5 & - & 2,5 \\
Tumor de Wilmes & 1 & 2,5 & - & - & - & - & 1 & 2,5 \\
Schannoma Maligno & - & - & 1 & 2,5 & - & - & 1 & 2,5 \\
Histiocitose Maligna & 1 & 2,5 & - & - & - & - & 1 & 2,5 \\
\hline TOTAL & 16 & 40,8 & 17 & 43,5 & 6 & 15,4 & 39 & 100 \\
\hline
\end{tabular}


Se reunirmos os 3 tipos de leucemia: Leucemia Linfóide Aguda (L.L.A.), Leucemia Mielóide Aguda (L.M.A.) e Leucemia Mieloide Crônica (L.M.C.), conforme demonstrado na Tabela I, o total perfaz $56,3 \%$ dos diagnósticos. A seguir, por ordem de importância numérica encontramos a doença de Hodgkin, rabdomiossarcoma, sarcoma de Ewing, neuroblastoma, retinoblastoma e tumor de Wilms.

Comparando os dados relativos a distribuição mundial do câncer em crianças $^{4}$, com os obtidos no presente estudo, constatamos que em ambos a leucemia aparece em primeiro lugar.

Dados deste estudo divergem do de BRUMINI et al $^{9}$ referente a levantamento no Brasil (1982), no que diz respeito a distribuição percentual das localizações de câncer em crianças menores de 14 anos de idade, uma vez que em primeiro lugar estes autores encontraram os tumores de gânglios linfáticos com $28.9 \%$ vindo na seqüência, por ordem de freqüência, aqueles localizados no sistema nervoso, ossos e articulações, olho, rim e pelve, tecidos moles, pele, gônodas, sistema hematopoético e outras localizações. Estes dados foram coletados em laboratórios de anatomia patológica e os exames citohematológicos e mielogramas não foram incluídos, o que pode explicar o baixo percentual das localizações em sistema hematopoético encontrada pelos autores acima citados.

Analisando os 22 casos de leucemia, verificamos que $95.4 \%$ é aguda, dado este semelhante ao da literatura $a^{3,5}$. Entre as leucemias agudas, encontramos para o tipo linfóide (L.L.A.) uma maior freqüência (62\%) e para o miel6ide (L.M.A.) uma freqüência menor (38\%). Na literatura consultada, até $80 \%$ dos casos são de L.L.A. e $20 \%$ L.M.A. ${ }^{3,13}$.

A maioria dos casos de leucemia linfóide aguda, neuroblastoma, tumor de Wilms, retinoblastoma e tumores hepáticos primários ocorre na população abaixo de 5 anos. Os tumores de Hodgkin e não Hodgkin e os de testículo e ossos ocorrem em maior freqüência em crianças maiores de $10 \operatorname{anos}^{2}$. Os dados por nós obtidos demonstram que os cânceres acometem com maior freqüência crianças menores de 10 anos de idade (84.5\%), comportando-se de forma bastante semelhante nos grupos etários de $0 \quad 5$ e 510 anos.

Dos casos por nós analisados, 11 (28\%) foram a óbito. Dentre estes, 10 $(90,9 \%)$ tiveram como diagnóstico principal a leucemia, sendo 6 na forma linfóide aguda e 4 na mielóide aguda.

Os dados referentes a procedência, estão descritos na Tabela II. Como pudemos observar, mais de $60 \%$ da população de estudo é proveniente de outras regiões do Estado de São Paulo e de outros estados do país. Este fato mostra que o Hospital das Clínicas da Faculdade de Medicina de Ribeirão Preto-USP é centro de referência, não apenas para a região mas também para outras regiões e estados, o que evidencia sua importância na assistência terciária. 
Tabela II - Distribuição de pacientes portadores de câncer, menores de 15 anos de idade, internados na Clínica Pediátrica do HCFMRP-USP, segundo a procedência, no período de $01 / 01$ a $31 / 12 / 87$.

\begin{tabular}{lcc}
\hline \multicolumn{1}{c}{ Procedência } & PACIENTES & \multicolumn{1}{c}{ No $^{\circ}$} \\
\hline Ribeirão Preto (Zona rural e urbana) & 7 & 17,9 \\
Outras cidades do SUS (ERSA-50) & 6 & 15,3 \\
Outras cidades do Estado de São Paulo & 15 & 38,4 \\
Outros Estados (MG. MT. GO) & 11 & 28,2 \\
\hline TOTAL & 39 & 100 \\
\hline
\end{tabular}

A Tabela III evidencia o diagnóstico segundo o sexo. Observamos a freqüência de câncer maior no grupo masculino $(71.3 \%)$ que no feminino $(27.8 \%)$, o mesmo ocorrendo com patologias tais como a leucemia linfóide aguda e a doença de Hodgkin, resultados estes semelhantes ao da literatura ${ }^{2}$.

TABELA III - Distribuição do diagnóstico de câncer em crianças menores de 15 anos de idade, internadas na Clínica Pediátrica do HCFMRP-USP, segundo sexo, no periodo de $01 / 01$ a $31 / 12 / 87$.

\begin{tabular}{|c|c|c|c|c|c|c|}
\hline \multirow{2}{*}{$\begin{array}{l}\text { SEXO } \\
\text { Diagnóstico }\end{array}$} & \multicolumn{2}{|c|}{ Masculino } & \multicolumn{2}{|c|}{ Feminino } & \multicolumn{2}{|c|}{ total } \\
\hline & $\mathrm{n}^{\circ}$ & $\%$ & $\mathbf{n}^{\circ}$ & $\%$ & $n^{\circ}$ & $\%$ \\
\hline Leucemia Línfóide Aguda (LLA) & 11 & 28,2 & 2 & 5,1 & 13 & 33,3 \\
\hline Leucemia Mielóide Aguda (LMÁ) & 6 & 15,3 & 2 & 5,1 & 8 & 20,5 \\
\hline Leucemia Mielóide Crônica (LMC & 1 & 2,5 & - & - & 1 & 2,5 \\
\hline Doença de Hodgkin & 3 & 7,6 & - & - & 3 & 7,6 \\
\hline Rabdomiossarcoma & 2 & 5,1 & 1 & 2,5 & 3 & 7,6 \\
\hline Neuroblastoma & 2 & 5,1 & - & - & 2 & 5,1 \\
\hline Sarcoma de Ewing & 1 & 2,5 & 1 & 2,5 & 2 & 5,1 \\
\hline Hepatoblastoma & - & - & 2 & 5,1 & 2 & 5,1 \\
\hline Tumor de Ovário & - & - & 1 & 2,5 & 1 & 2,5 \\
\hline Tumor de Wilms & 1 & 2,5 & - & - & 1 & 2,5 \\
\hline Retinoblastoma & - & - & 1 & 2,5 & 1 & 2,5 \\
\hline Schwannoma Maligno & - & - & 1 & 2,5 & 1 & 2,5 \\
\hline Histiocitose Maligna & 1 & 2,5 & & - & 1 & 2,5 \\
\hline TOTAL & 28 & 71,3 & 11 & 27,8 & 39 & 100 \\
\hline
\end{tabular}

Vale ressaltar que o número de internações por pacientes variou. Alguns, tiveram internações única e longa, outros, várias e curtas, geralmente durante os ciclos de quimioterapia. Isto ocorreu porque os pacientes, que apresentavam condições físicas satisfatórias e disponibilidade para ir e vir ao hospital diariamente, permaneciam internados somente durante o período de quimioterapia, com alta-licença a seguir, retornando no dia seguinte para a continuidade do tratamento. 
O tratamento das doenças neoplásicas malignas é feito basicamente pela quimioterapia, radioterapia e cirurgia. A terapêutica instituída varia com o tipo de neoplasia, utilizando-se associações como: quimioterapia e radioterapia, cirurgia e radioterapia, quimioterapia e cirurgia; cirurgia, quimioterapia e radioterapia. No nosso estudo observamos que em associação ou isoladamente a quimioterapia foi o tratamento mais freqüente.

\section{A AMPLIAÇÃO DO MODELO CLÍNICO: DA DOENÇA CÂNCER À CRIANÇA COM CÂNCER E SUA FAMÍLIA.}

$\mathrm{Na}$ assistência à criança com câncer predomina o atendimento clínico, individual, curativo, centrado na área hospitalar, onde existe um aparato altamente sofisticado e uma grande intervenção artificial sobre a natureza dos corpos. A enfermagem é parte integrante deste processo de trabalho e para apreender a criança e atender às suas necessidades, ela reproduz e detalha o conhecimento morfo-fisio-patológico utilizando o mesmo como instrumental para intervir a nível individual.

A prática de saúde baseada neste modelo no qual a patologia, o diagnóstico e a terapêutica são os focos principais, mostrou-se insuficiente para atender as necessidades apresentadas pelas crianças com câncer e suas famílias atendidas na Unidade de Pediatria do Hospital das Clínicas da Faculdade de Medicina de Ribeirão Preto da Universidade de São Paulo.

O objetivo da assistência sofreu transformações com o aumento da demanda, a perspectiva de cura para um grande número de crianças devido ao desenvolvimento de novas modalidades terapêuticas, de novos métodos de investigação clínica e da deteç̧ão precoce da doença maligna com o conseqüente aumento da sobrevida. Numa perspectiva mais atual, a meta é tentar proporcionar uma melhor qualidade de vida, levando em consideração a integralidade da criança, atendendo aos aspectos para além da patologia.

Desta forma, a assistência de enfermagem caracteriza-se por atividades técnicas, centrada no anátomo-fisiológico e ações de caráter mais amplo, que visam a manutenção do crescimento e desenvolvimento, procurando proporcionar uma melhor qualidade de vida. Além das ações básicas referentes a higiene, alimentação e medicação, soma-se outras como recreação e implementação do relacionamento mãe-filho através do alojamento conjunto.

O objeto de trabalho já não é mais apenas o corpo biológico e, sim, a criança com câncer em toda sua especificidade, cujas necessidades, antes restritas a cura da doença, agora, são determinadas pela criança doente, respeitando seu crescimento, desenvolvimento e sua individualidade. $O$ processo terapêutico não se restringe mais a restauração do funcionamento anátomo-fisiológico do corpo humano. A finalidade é proporcionar além desta 
restauração, a qualidade de vida durante o crescimento e desenvolvimento da criança, preservando suas relações sociais, afetivas e psicológicas. O modelo clínico, portanto, ampliou-se.

Para responder a esta nova necessidade, os instrumentos ou meios de trabalho também alteram-se. Novas ações e abordagens de enfermagem são acrescentadas as já existentes utilizando-se outros conhecimentos além da Anatomia, Fisiologia e Patologia. Sendo assim, a enfermagem vai buscar no referencial térico da Psicologia, Sociologia, Antropologia e Epidemiologia conhecimentos para responder as necessidades dessa criança doente e sua família.

A literatura aponta que para assistir a criança hospitalizada, diferentes abordagens e correntes que compõem a área de conhecimento em Psicologia estão sendo utilizadas. Atualmente, embora de forma não sistemática, alguns conhecimentos da Epidemiologia instrumentalizam o trabalho da enfermeira pois possibilitam a organização da clínica, uma vez que, através dos índices de incidência, prevalência e mortalidade ela planeja número e rotatividade de leitos, aperfeiçoamento de técnicas, distribuição de leitos segundo sexo, idade e patologia e ainda, educação continuada para o atendimento do conjunto de crianças doentes.

Em relação à criança com câncer atendida na clínica pediátrica do hospital em estudo, o atual recorte intelectual é o olhar sobre um ser em crescimento e desenvolvimento, que necessita de condições adequadas de higiene, alimentação e recreação além de ações para recuperar seu organismo biológico.

O conhecimento psicológico, de relações humanas e sociais, é trazido para a enfermagem através da equipe multiprofissional e existe uma tentativa de relacioná-los numa mesma prática, uma vez que teoricamente esta relação está descrita como possível. Entretanto, se na teoria é possível um exercício de síntese, para apreender a criança com câncer através destas áreas do conhecimento, na prática is to não é concretizado de imediato.

O trabalho concreto da equipe multiprofissional, através do estudo de casos fornece elementos para atuação conjunta da equipe - médico, enfermeira, psicóloga, assistente social e nutricionista. Assim, uma nova ordem instalase, uma vez que a responsabilidade da assistência não $e$ mais apenas do profissional médico e da enfermeira.

Enquanto o médico manipula mais os conhecimentos relativos ao diagnóstico e terapêutica, o psicólogo, os afetivos e emocionais e o terapêuta ocupacional os de lazer e recreação, a enfermeira, que é o profissional que fica junto à criança em momentos críticos, como nas intervenções, nos cuidados higiênicos, nas comunicações com pais e familiares, é a que tem os elementos para fazer a síntese desta assistência. Portanto, ela está saindo de uma função essencialmente técnica, para assumir a função sintetizadora da assistência à 
criança com câncer e sua família, sem assumir a responsabilidade de ser um profissional onipotente.

\section{CONSIDERAÇÕES FINAIS}

A assistência à criança com câncer na clínica em questão adquiriu algumas cracterísticas que já podem ser percebidas no dia a dia: o ambiente hospitalar tornou-se mais informal, descontraído, a mãe veio para dentro do hospital e tem participado ativamente do cuidado a seu filho, as rotinas estão menos rígidas, o relacionamento entre criança, família e equipe de saúde tem ocorrido de forma menos tensa, a equipe está atenta aos agravos psíquicos, de desenvolvimento e crescimento que a criança pode apresentar. Para a equipe de saúde abre-se a perspectiva de uma assistência integral à criança.

E nesse processo contínuo, numa realidade concreta, levando em consideração a especificidade do momento, equipe e cliente que se consegue a transformação do processo de trabalho e uma assistência qualitativamente melhor. Assim, concluímos que a assistência à criança com câncer desenvolvida na Unidade de Pediatria do Hospital das Clínicas de Ribeirão Preto está em fase de transformação, saindo do modelo exclusivamente clínico e caminhando para outro, que tem algumas características de ampliação do referencial teórico e nova instrumentalização.

LIMA, R.A.G. de et al. Assistance to children with cancer: analysis of the work process.

Rev.Eac.Enf.USP, v.30, n.1, p.14-24, Apr. 1996.

This study tries to analyse some principle of the working process in the assistance of children with cancer, based on the Model of the Technological Organization of Work. It started from a theoretical principle that nursing is a social practice and so is historically structured, socially articulated, and so nursing is part of the collective process of work. We pointed out toward the fact that the process of the nursing work in assisting a hospitalized child, that it is under a transformation phase, going through the exclusively clinic model to another one, which has some characteristics of amplification to the theoretical reference and a new instrumentalization.

UNITERMS: Neoplasms-child. Nursing Care. 


\section{REFERÊNCIAS BIBLIOGRÁFICAS}

01. ALERT, S.J.; LONGCHONG, R.M. Incidencia y mortalidade de las neoplasias malígnas de los niños em Cuba. Rev. Cubana Pediatr., v.51, p. 31-40, 1979.

02. ALTMAN, A.J. Simpósio sobre oncologia pediátrica.Clín. Pediatr. Am. Norte, v.3, p. 551$891,1985$.

03. ANDREA, M.L.M. Leucemias e linfomas. J. Pediatr., v.55, n.2, p. 166-74. 1983.

04. ANTONIELLI, C.B.G. Possibilidades atuais de cura da criança com câcer. Rev. Paul. Pediatr., v.6, n.22, p. $102-4,1988$.

05. AUR, J.A.; BRANDALISE, S.R. Leucemia linfocítica aguda: aspectos atuais. Rev. Paul. Pediatr., v. 1, n.5, p. 34-48, 1983.

06. BANCO MUNDIAL. Brasil: novo desafio à saúde do adulto. Washington, 1991. (Mimeografado)

07. BRASIL. Ministério da Saúde. Instituto Nacional do Câncer. Coordenação de Controle do Câncer (PRO-ONCO). O problema do câncer no Brasil. Rio de Janeiro, 1992.

08. BRASIL . Ministério da Saúde. Previdencia Social. Instituto Nacional de Previdencia Social. Classificação internacional de doenças, lesões e óbito: revisão 9. São Paulo. Centro Brasileiro de Classificação de Doenças em Português. 1979.

09. BRUMINI, R. et al. Cancer no Brasil: dados histopatológicos. 1976 - 1980. Rio de Janeiro, Ministério da Saúde. Campanha Nacional de Combate ao Câncer, 1982.

10. GONÇALVES, R.B.M. Tecnologia e organização das práticas de saúde: características tecnológicas do processo de trabalho na Rede Estadual de Centros de Saúde de São Paulo. São Paulo, 1986. 399p. Tese (Doutorado). Faculdade de Medicina, Universidade de São Paulo.

11. LIMA, R.A.G.; ROCHA, S.M.M.; TONE, L.G. O câncer infantil: caracterização da população atendida em um hospital - escola como subsídios para a assistência de enfermagem. Medicina, v.26, n.4, p.627-35, 1993.

12. MENDONÇA, G.A.S. Câncer no Brasil: um risco crescente. Rev. Bras. Cancerol., v.38, n.4, p. $167-76,1992$.

13. SABINO, M.A. et al. Leucemia linfoncítica aguda na infância: conceitos atuais no tratamento e experiências do Instituto da Criança. Pediatr.,v.3, n.4, p. 296-303, 1981.

14. SEADE. Sistema Estadual de Análise de Dados. São Paulo, Bol. Inf., 1988.

15. VOUTE, P.A. Câncer na infância. In: UICC (Uniāo Internacional Contra o Câncer). Manual de Oncologia Clínica. 5. ed. São Paulo, Fundação Oncocentro de São Paulo. p. 383-99. 